\title{
Overexpression of aspen sucrose synthase gene promotes growth and development of transgenic Arabidopsis plants
}

\author{
Fuyu $\mathrm{Xu}^{1}$, Chandrashekhar P. Joshi ${ }^{1,2}$
}

\footnotetext{
${ }^{1}$ Biotechnology Research Center School of Forest Resources and Environmental Science, Michigan Technological University, Houghton, USA;

${ }^{2}$ Department of Bioenergy Science and Technology, Chonnam National University, Buk-Gu, Gwangju, Korea.

Email: cpjoshi@mtu.edu
}

Received 14 September 2010; revised 22 October 2010; accepted 29 October 2010.

\section{ABSTRACT}

In plants, sucrose synthase (SUS) enzymes catalyze conversion of sucrose into fructose and UDP-glucose in the presence of UDP. To investigate the impact of overexpression of heterologous SUS on the growth and development of Arabidopsis, we transformed Arabidopsis plants with an overexpression vector containing an aspen SUS gene (PtrSUS1). The genomic PCR confirmed the successful integration of PtrSUS1 transgene in the Arabidopsis genome. PtrSUS1 expression in transgenic Arabidopsis plants was confirmed by RT-PCR. The SUS activity was dramatically increased in all transgenic lines examined. The three selected transgenic PtrSUS1 lines exhibited faster growth and flowered about 10 days earlier compared to untransformed controls, and also possessed 133\%, 139\%, and 143\% SUS activity compared to controls. Both fresh weights and dry biomass yields of the whole plants from these three selected transgenic lines were significantly increased to $125 \%$ of the controls. Transgenic PtrSUS1 lines also had a higher tolerance to higher concentration of sucrose which was reflective of the increased SUS activity in transgenic versus wild-type plants. The growth differences between wild-type and transgenic plants, either in root and hypocotyl length or in fresh and dry weight of whole plant, became more pronounced on the media containing higher sucrose concentrations. Taken together, these results showed that the early flowering, faster growth and increased tolerance to higher sucrose in transgenic lines were caused by the genome integration and constitutive expression of the aspen PtrSUS1 gene in transgenic Arabidopsis.

Keywords: Arabidopsis; Aspen Trees (Populus Tremuloides); Over-Expression; Sucrose Synthase
(SUS); SUS Enzyme Activity

\section{INTRODUCTION}

Sucrose synthase (SUS) is an important enzyme in carbohydrate metabolism of plants that catalyzes a reverseble reaction of converting sucrose into fructose and UDP-glucose in the presence of UDP. SUS, therefore, mainly functions in tissues that actively metabolize sucrose [1-3]. The level and distribution of SUS activity in different tissues provides a meaningful insight into the transport and consumption of carbohydrates during the plant development and plant response to various environmental stresses $[4,5]$. SUS activity has been localized in different intracellular structures, such as cell membranes [6,7], the cytoskeleton [8], and the tonoplast [9]. SUS also exists in two forms, the soluble form in the cytosol and the second form in association with the plasma membrane or cell walls $[10,11]$, with the latter proposed to be involved in the synthesis of cell wall components by providing UDP-glucose directly to the cellulose synthases and callose synthases [12]. Using differentiating tracheary elements as a model system, Salnikov et al. [13] and Salnikov et al. [14] showed that the SUS was specifically localized in and around the plasma membranes and the microtubules during the secondary wall thickenings, and thus establishing a spatial relationship and context with secondary wall cellulose synthesis. SUS enzyme activity was localized in roots of wheat in response to hypoxic conditions, and was linked with the secondary wall thickenings and the deposition pattern of cellulose $[15,16]$. SUS activity has also been positively linked with sink strength in storage organs of potatoes [17], tomato fruits [18], developing cotton seed [19], maize kernels [20,21], and pea embryos [22,23].

The involvement of SUS in plant growth and reproductive development was investigated by several re- 
search groups. The over-expression of cotton SUS in tobacco transgenic plants increased the total biomass amount [24]. While studying the activities of enzymes involved in the control of carbon flux of two genotypes of Vigna radiata (mung bean) differing in seed weight and size, Chopra et al. [25] found that the change of SUS activities was positively correlated with the biomass accumulation of seeds in two genotypes, i.e., the large-seed genotype maintained high SUS activity longer, thus with the longer seed filling phase and stronger sink compared to the small seeds. It has also been reported that SUS cooperates with UGPase and AGPase to control the efficient partitioning of sucrose into ADP glucose and thereby regulate the seed sink strength in the mung bean plants $[26,27]$. During the flower bud formation, greater activities of SUS and other sugar catabolizing enzymes may enhance the capacity of buds to attract assimilates, thus accelerating bud growth and increasing the number of bud primordia [28]. It has recently been reported that AtSUS from Arabidopsis showed specific roles in seed and silique development [5]. Overexpression of the cotton SUS gene in hybrid poplar promoted $2 \sim 6 \%$ of cellulose content and increased crystallinity in all transgenic lines with the increased SUS enzyme activity compared to controls [29]. However, the overexpression of the same cotton SUS construct into tobacco did not change the cellulose percentage [24]. The difference between transgenic tobacco and transgenic hybrid poplar plants is probably due to the less secondary growth in tobacco compared to poplars.

The role of SUS in flowering process has also been examined using antisense SUS constructs in transgenic tomato (Lycopersicon esculentum) plants, in which SUS activity was severely inhibited by the antisense RNA in flowers and fruit pericarp tissues, and in turn causing a reduced fruit set and a slower growth rate of fruits [30]. The dramatically decreased sucrose unloading capacity of 7-day-old fruit was observed in antisense lines with low SUS activity, accompanied by a slower growth rate of antisense fruit from the first week of flowering and a reduced fruit set. These results suggest that SUS participates in regulating sucrose import capacity of young tomato fruit, which is a determinant for fruit set and development. SUS gene expression was dramatically reduced in transgenic cotton plants expressing a SUS cosuppression construct, which caused the reduced number of fiber initiations and reduced fiber length, and induced numerous collapsed fiber cells, and even resulted in the failure of setting of seeds in some lines [31]. These results also supported a specific role for SUS during fiber initiation and elongation in cotton.

The release of poplar genome and availability of poplar EST/cDNA libraries provided excellent platform to investigate the function of SUS family in plant growth and development. The results of genome-wide transcript profiling have shown that SUS1 and SUS2 were the most abundant transcripts among poplar SUS family members (Xu and Joshi, submitted). In addition, PttSUS1 and PttSUS2 from Populus tremula (L.) $\times$ tremuloides (Michx.) were also shown to dramatically increase during tension wood (TW) formation, supporting the significant role of SUS in cellulose synthesis in TW [32]. Previously, our lab successfully cloned PtrSUS1 and PtrSUS2 from aspen trees (Populus tremuloides Michx.), and we also found that they were up-regulated in developing xylem of TW (Joshi et al., unpublished data). So we were interested to investigate the effects of overexpression of heterologous PtrSUS in Arabidopsis. In this study, we show the successful integration of aspen SUS gene (PtrSUS1) in Arabidopsis genome by genomic PCR, overexpression of PtrSUS1 genes in Arabidopsis through RT-PCR, and increased SUS enzyme activities in transgenic plants, suggesting that the observed faster growth during early developmental stages and early flowering in transgenic lines were caused by the integration and overexpression of the aspen SUS gene in the Arabidopsis genome.

\section{MATERIALS AND METHODS}

\subsection{Plant Materials}

Arabidopsis thaliana wild-type (Col-0), SUS overexpressing transgenic lines and pBI121 (containing 35S-GUS-NOS construct) transgenic control lines were grown at $22^{\circ} \mathrm{C}$ under $16 \mathrm{~h}: 8 \mathrm{~h}$ light:dark conditions with approximately $200 \mu \mathrm{m}$ photons $\mathrm{m}^{-2} \mathrm{sec}^{-1}$ light intensity either on Arabidopsis soil mix (Lehle Seeds, Round Rock, TX, USA) or agar plates.

\subsection{DNA Constructs and Plant Transformation}

The PtrSUS1 overexpression construct was prepared by Drs. Takeshi Fujino and Suchita Bhandari in our laboratory. PCR was performed with the following primers using a full-length PtrSUS1 cDNA of $2.84 \mathrm{~kb}$ (GenBank accession\#: AY341026) as a template. The forward primer was Susy_F_SpeI (5'-CGACTAGTGGCATTAAACTTAAGGAGC-3'); the reverse primer was Susy R SmaI (5'-CGCCCGGGCAAACCAACCCATG TTCC $-\overline{3}$ '). The PCR product was first cloned in PCR2.1 Topo vector (Invitrogen) and digested with SpeI and SmaI, and the insert was cloned into the binary plant vector pBI121 (Clontech, San Diego, CA, USA) digested with XbaI (SpeI compatible) and Ecl136II (blunt), between the cauliflower mosaic virus $35 \mathrm{~S}$ promoter (35SP) and Nopaline synthase terminator (NOST). The resultant plasmid, named 35S-PtrSuS1-NOST, was mo- 
bilized to Agrobacterium tumefaciens strain C58 and used for plant transformation. Arabidopsis thaliana wild-type (Col-0) plants were transformed with the construct by the Agrobacterium-mediated floral dip method [33], and transformed seeds were surface-sterilized, sown and selected on $0.5 \mathrm{x}$ MS-medium containing $1 \%$ $(\mathrm{w} / \mathrm{v})$ sucrose and $100 \mu \mathrm{g} \mathrm{ml}^{-1}$ kanamycin in Petri dishes. Transgenic seeds were germinated and grown in tissue culture room at $24 \pm 2^{\circ} \mathrm{C}$ under a 16-h light/8-h dark illumination regime with about $150 \mu \mathrm{mol} \mathrm{m} \mathrm{m}^{-2} \mathrm{sec}^{-1}$ fluorescent illumination in daylight setting. Kanamycin-resistant seedlings $\left(T_{1}\right)$ were transplanted into soil and grown to maturity for seed harvesting. $T_{2}$ seeds were collected from selfed T1 plants, and a portion of the $T_{2}$ seeds was germinated on $0.5 \mathrm{x}$ MS medium with kanamycin selection and transplanted into soil. $\mathrm{T}_{3}$ seeds were harvested from $T_{2}$ plants and used in this study.

\subsection{PCR Confirmation of PtrSUS1 Transgene Integration in Arabidopsis}

Genomic DNA was extracted from the leaves of transgenic Arabidopsis seedlings of $\mathrm{T}_{1}, \mathrm{~T}_{2}$, and $\mathrm{T}_{3}$ generations grown in soil after selection on $0.5 x$ MS medium containing Kanamycin. Twelve independent transgenic lines were produced and confirmed in this study. Transgenic nature of selected lines was confirmed by three pairs of primers: $1^{\text {st }}$ pair containing a forward primer from $35 \mathrm{~S}$ promoter sequence (35SP-F) and a reverse internal primer from PtrSUS1 (SusyInR); $2^{\text {nd }}$ pair containing an internal forward primer derived from PtrSUS1 (SusyInF) and a reverse primer (NostR) from the NOST; $3^{\text {rd }}$ pair containing SusyInF and SusyInR primers.

\subsection{Growth of Transgenic Arabidopsis Lines on Media with Varying Sucrose Concentrations}

Sterilized seeds of wild type and T3 transgenic Arabidopsis lines were cultured on $0.5 \mathrm{x}$ MS medium without sucrose in Petri dishes, vertically placed in racks, for 3 days, and were transplanted onto $0.5 \mathrm{x}$ MS medium with $0,1,2,3,4,6,8 \%$ sucrose to grow either in regular light condition or wrapped with aluminum foil for dark treatment. Each plate contained 15 wild-type or PtrSUS1 transgenic plants. Root and hypocotyl lengths were measured on day 7 day after the transfer. Average length was taken from one set of 10 plants and three replicate plates per treatment were used for each transgenic line. The fresh weight of each individual whole plant was measured immediately after the harvest. Biomass yield (dry weight in $\mathrm{mg}$ ) was recorded after drying in an oven to practically zero per cent moisture content roughly after $48 \mathrm{~h}$ at $70^{\circ} \mathrm{C}$. Whole plants were harvested for biomass measurements and total protein extraction after 3 weeks of transfer.

\subsection{RNA Preparation and Semi-Quantitative RT-PCR}

Seedlings were harvested after different periods of incubation on serial concentrations of sucrose. Total RNA was isolated by using RNeasy Plant Mini Kit (Qiagen, Valencia, CA, USA), and treated with DNase I (NEB, MA, USA). PtrSUS1 transcripts in transgenic Arabidopsis plants were determined by RT-PCR. $1 \mu \mathrm{g}$ of total RNA was used as a template for first-strand cDNA synthesis with SuperScript ${ }^{\circledR}$ II RT kit (Invitrogen). The synthesis of the first strand cDNA was conducted at $42^{\circ} \mathrm{C}$ for $30 \mathrm{~min}$ followed by $70^{\circ} \mathrm{C}$ for $10 \mathrm{~min} .2 \mu 1$ of RT reaction (cDNA) which is equivalent to $100 \mathrm{ng}$ of RNA was used in the reaction of $25 \mu \mathrm{l}$ with $\mathrm{GoTaq}^{\circledR}$ Green PCR Master Mix (Promega, Madison, WI). In order to differentiate PtrSUS1 expression from the endogenous AtSUS gene expression, we designed PtrSUS1 genespecific primer pairs using six AtSUS cDNA sequences as references while using PRIMER3 software (http:// frodo.wi.mit.edu/cgi-bin/primer3/primer3 www.cgi). Primers were purchased from Sigma (St. Louis, MO). The following primers were used: for PtrSUS1 gene, PtrSUS1-F (5'-CAT CCC CCG AAT TCT CAT TA-3'), PtrSUS1-R (5'-TCC ACA AGT GGT TCC TAC TGC-3'); for ubiquitin gene (At4g05320), AtUBQ-F (5'-GAA TCC ACC CTC CAC TTG GTC-3'), and AtUBQ-R (5'-CGT CTT TCC CGT TAG GGT TTT-3'). PCR reaction was conducted at $94^{\circ} \mathrm{C}$ for $10 \mathrm{~min}$, then 40 cycles of $94^{\circ} \mathrm{C}$ for $30 \mathrm{sec}, 60^{\circ} \mathrm{C}$ for $30 \mathrm{sec}$, and $72^{\circ} \mathrm{C}$ for $1 \mathrm{~min}$, followed by an additional extension of $72^{\circ} \mathrm{C} 10 \mathrm{~min}$.

\subsection{In Situ Staining of Sucrose Synthase Activity}

Arabidopsis seedlings were prepared according to Sergeeva et al. [34] with some modifications. Briefly, soaked seeds were grown in Petri dishes with two layers of moist filter paper and placed at $4{ }^{\circ} \mathrm{C}$ for stratification in darkness for 2 days. They were then transferred to $23^{\circ} \mathrm{C}$ in the light with a $16 \mathrm{~h}: 8 \mathrm{~h}$ day:night regime and grown for $7 \mathrm{~d}$. Roots from poplar were obtained from tissue culture as described above. In situ staining and localization of sucrose synthase activity were conducted by incubating whole plants or roots in a reaction medium as described earlier [34-36] with some modifications. The incubation medium contained $100 \mathrm{mM}$ HEPES-NaOH buffer (pH 7.4), $10 \mathrm{mM} \mathrm{MgCl} 2,2$ mM EDTA, $0.2 \%$ BSA, $2 \mathrm{mM}$ EGTA, $1 \mathrm{mM}$ NAD, $1 \mathrm{U}$ phosphoglucomutase (PGM) from rabbit muscle, $1 \mathrm{U}$ glucosephosphate dehydrogenase (G6PDH) from Leuconostoc mesenteroides, $20 \mu \mathrm{M}$ glucose-1,6-bisphosphate , 1 U UDPG-pyrophosphorylase (UGPase) from beef liver (Sigma, St. Louis, $\mathrm{MO}$ ), and $0.03 \% \mathrm{NBT}$, and the reaction was started by adding substrate solution (containing sucrose, UDP and 
PPi) resulting in final concentration of substrates in the incubation medium of $3.6 \mathrm{mM}, 71 \mu \mathrm{M}$, and $71 \mu \mathrm{M}$, respectively. Controls were incubated without sucrose, or without PGM, glucose-1,6-bisphosphate and PPi, or without NAD.

\subsection{Quantitative SUS Enzyme Assays}

A 0.5-1.0 g sample of leaves or whole plants was grounded in liquid nitrogen with a mortar and pestle. Extraction buffer contained $50 \mathrm{~mm}$ HEPES-KOH ( $\mathrm{pH}$ 7.4), $1 \mathrm{~mm} \mathrm{MgCl}_{2}, 1 \mathrm{~mm}$ EDTA, $1 \mathrm{mM} \mathrm{EGTA,} \mathrm{10 \%} \mathrm{(v/v)}$ glycerol, $0.1 \%(\mathrm{w} / \mathrm{v})$ bovine serum albumin (BSA), 5 $\mathrm{mM}$ DTT, $20 \mathrm{mM} \beta$-mercaptoethanol, 1\% (w/v) insoluble PVP-40 (polyvinylpyrrolidone), and $0.1 \mathrm{mM}$ PMSF (phenylmethylsulfonyl fluoride), $1 \mu \mathrm{g} / \mathrm{ml}$ pepstatin, and $2 \mu \mathrm{g} / \mathrm{ml}$ leupeptin. The ratio of powdered tissue to extraction buffer was about $5 \mathrm{ml} / \mathrm{g}$ fresh weight. The homogenate was centrifuged at $4^{\circ} \mathrm{C}$ at $13,000 \mathrm{~g}$ for $5 \mathrm{~min}$. The supernatant was collected and equilibrated with 50 mm Hepes-NaOH (pH 7.5), 1 mm EDTA and 1 mm DTT. Protein extract concentrations were determined using a Bio-Rad protein assay kit (Bio-Rad, Hercules, CA) with BSA as the standard.

Quantitative sucrose enzyme activity assays were carried out in the direction of sucrose cleavage according to $\mathrm{Xu}$ et al. [35], Zrenner et al. [17] and Ruan et al. [31] with minor modifications, and UDP-glucose from sucrose was measured spectrophotometrically by the absorption change at $340 \mathrm{~nm}$ molar extinction $\left(A_{340}\right)$ as NAD was reduced to NADH by UDP-Glc dehydrogenase (UDPG-DH; Sigma, St. Louis, Mo.). Briefly, each assay was carried out with 30-40 $\mu$ l of crude extract equivalent to about $40 \mu \mathrm{g}$ total protein in $250 \mu \mathrm{l}$ reaction mixtures containing $20 \mathrm{mM}$ HEPES-KOH (pH 7.0), 100 $\mathrm{mM}$ sucrose and $4 \mathrm{mM}$ UDP. A blank without sucrose and UDP was included to subtract the background. Incubation was carried out at $25^{\circ} \mathrm{C}$ for $40 \mathrm{~min}$ and stopped by boiling for $5 \mathrm{~min}$. The determination of UDP-glucose was conducted in $200 \mathrm{mM}$ glycine- $\mathrm{NaOH}$ ( $\mathrm{pH} 8.9$ ), 5 $\mathrm{mm} \mathrm{MgCl}_{2}, 2 \mathrm{~mm}$ NAD and 0.04 units of UDP-glucose dehydrogenase, and the reaction mixture was incubated for $30 \mathrm{~min}$ at $37^{\circ} \mathrm{C}$ before measurement of $\mathrm{A}_{340}$. Blanks without sucrose and UDP were included to subtract the background.

\section{RUSULTS}

\subsection{Generation and Confirmation of PtrSUS1 Transgenic Arabidopsis Plants}

PtrSUS1, a full-length aspen SUS cDNA (GenBank accession \# AY341026) was successfully cloned into the binary vector pBI121 replacing the GUS gene (Figure 1). Agrobacterium-mediated floral dip transformation me- thod was used to produce transformed seeds. Kanamycin-resistant plants were subjected to genomic PCR confirmation. Three pairs of primers were used to verify integration of the PtrSUS1 transgene in Arabidopsis as described in Materials and methods and all tested transgenic lines were found to carry the transgene. Figure 2(a) shows the results from one of the representative lines, and the successful transgenic events with intact PtrSUS1 cDNA integration show three fragments: $\sim 2 \mathrm{~kb}$ from 35SP-F::SusyInR, $\sim 1.2 \mathrm{~kb}$ from SusyInF::NostR, and 366 bp SusyInF::SusyInR primer pairs as expected. Three representative transgenic Arabidopsis lines, denoted as T3-6, T3-7, and T3-11 (Figure 2(c)), were selected for further analyses.

Under standard growth conditions in the greenhouse, transgenic lines were phenotypically indistinguishable from the wild type plants before inflorescence emergence. However, the speed of inflorescence development and timing of flowering from PtrSUS1 transgenic plants were much faster and earlier than wild-type plants (Figure 2(b) and Table 1).

\subsection{Overexpression of PtrSUS1 Transgene in Arabidopsis}

Semi-quantitative RT-PCR was performed to confirm the expression of the PtrSUS1 transgene in $\mathrm{T}_{3}$ plants from three homozygous transgenic lines. The total RNA was extracted from leaves of three transgenic lines and corresponding controls grown in soil and/or cultured in Petri dishes. Expression of an Arabidopsis ubiquitin gene was used as an internal control. Using PtrSUS1 gene-specific primers that can discriminate between as-

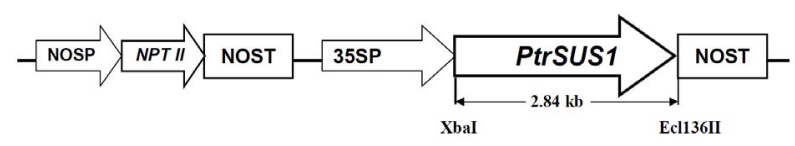

Figure 1. Structure of the 35S-PtrSUS1-NOST construct used for Arabidopsis transformation. To introduce the full-length PtrSUS1 cDNA into Arabidopsis genome by Agrobacteriummediated method, the XbaI-Ecl136II fragment of PtrSUS1 was inserted between the cauliflower mosaic virus $35 \mathrm{~S}$ promoter (35SP) and the nopaline synthase gene terminator (NOST) in a binary plant vector pBI121, named 35S-PtrSUS1-NOST. pBI121 carries the neomycin phosphotransferase II (NPTII) gene, driven by the nopaline synthase gene promoter (NOSP), for selection of transgenic cells by kanamycin.

Table 1. Time of flowering for wild type and transgenic plants.

\begin{tabular}{ccccc}
\hline Genotype & Col-0 & T3-6 & T3-7 & T3-11 \\
\hline $\begin{array}{c}\text { Days of first } \\
\text { flower buds visible }\end{array}$ & 23 & 15 & 13 & 14 \\
\hline
\end{tabular}

Note: Days, averaging day from date of sowing seeds in Petri dish, excludeing a 3-day stratification at $4^{\circ} \mathrm{C}$. 


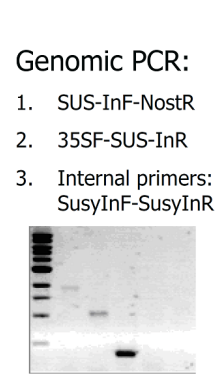

123

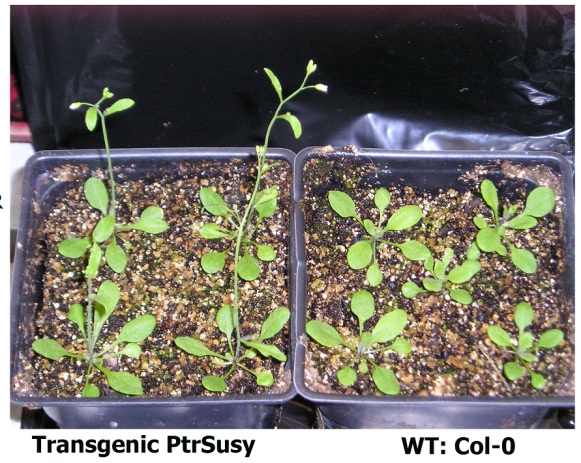

(b)

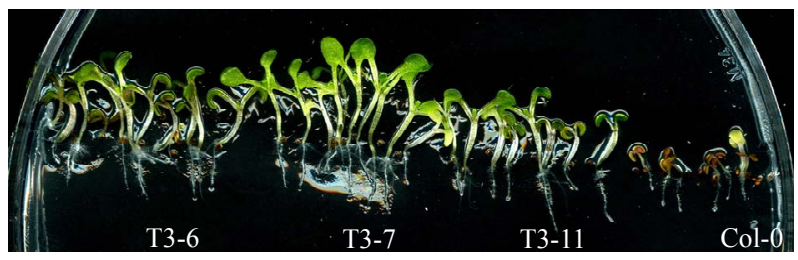

(c)

Figure 2. Genomic PCR confirmation and phenotypes of representative PtrSUS1 transgenic Arabidopsis. Three primer pairs were used to verify the PtrSUS1 transgenic Arabidopsis plants (a); 15 d-old seedlings from transgenic plants of one line and wild-type (Col-0) (b); Growth of three representative transgenic lines and wild-type in $0.5 \mathrm{x}$ MS medium containing $100 \mu \mathrm{g} / \mathrm{ml}$ kanamycin.

pen and Arabidopsis SUS genes, all three transgenic lines tested showed expression of the PtrSUS1 gene but it was absent in other control plants (Figure 3). Similar results were obtained by using plants grown in greenhouse and Petri dish samples since the PtrSUS1 gene is under the control of a constitutive $35 \mathrm{~S} \mathrm{CaMV}$ promoter.

\subsection{SUS Enzyme Activity}

We tested whether SUS enzyme activity was enhanced in transgenic lines using in situ staining, as was described previously for tissue sections [36,37]. Whole seedlings were fixed and incubated in the staining reaction buffer. Figure 4 shows the results from the roots of the wild type and transgenic plants but similar results were obtained using other tissues. Blue formazan color from reduced nitroblue tetrazolium (NBT) was observed, indicating enzyme activity. This blue precipitation specifically indicated SUS activity, as compared and determined from the absence of staining in the control reactions without sucrose. Transgenic lines showed stronger blue color, indicating stronger SUS enzymatic activity compared to wild-type untransformed controls (Figure 4).

Protein extracts of transgenic and wild type plants were further assayed for quantitative SUS enzyme activities by determining the rate of UDP-glucose production. SUS activities of three transgenic plants were 480 , 501, and $518 \mathrm{nmol}$ UDP-glucose per minute and per mg of protein, corresponding to $133 \%, 139 \%$, and $143 \%$ of wild type (361 nmol UDP-glucose per minute and $\mathrm{mg}$ protein) (Figure 5), and the difference between transgenic and wild type plants was statistically very highly or highly significant. This demonstrated that introducetion of aspen SUS gene PtrSUS1 resulted in significant increase of SUS activity in transgenic Arabidopsis

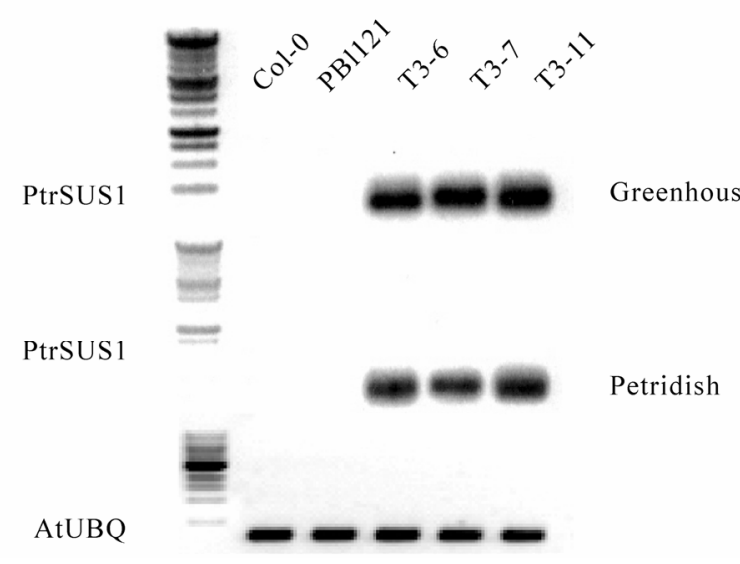

Figure 3. Overexpression of the PtrSUS1 gene in transgenic plants. Three-week-old seedlings of the wild-type (Col-0), GUS vector carrying control plants (pBI121: p35S-GUS) and three lines of transgenic plants overexpressing PtrSUS1 (p35S-PtrSUS1) were collected from both greenhouse and vertical Petri dishes were used for total RNA isolation and subsequent RT-PCR. Expression of an Arabidopsis ubiquitin gene was used as an internal control. PtrSUS1 Gene-specific primers as described in "Materials and methods" were used to determine the presence and level of PtrSUS1 transcript of transgenic plants.

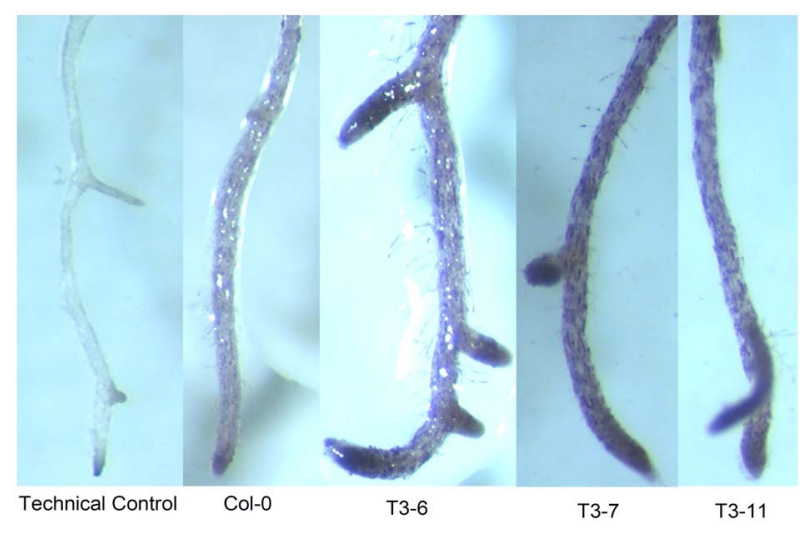

Figure 4. Sucrose synthase activity in 7-d-old roots of WT (Col-0) and three transgenic lines T3-6, T3-7 and T3-11. Stained roots from whole plant staining including one technical reaction control without sucrose. 


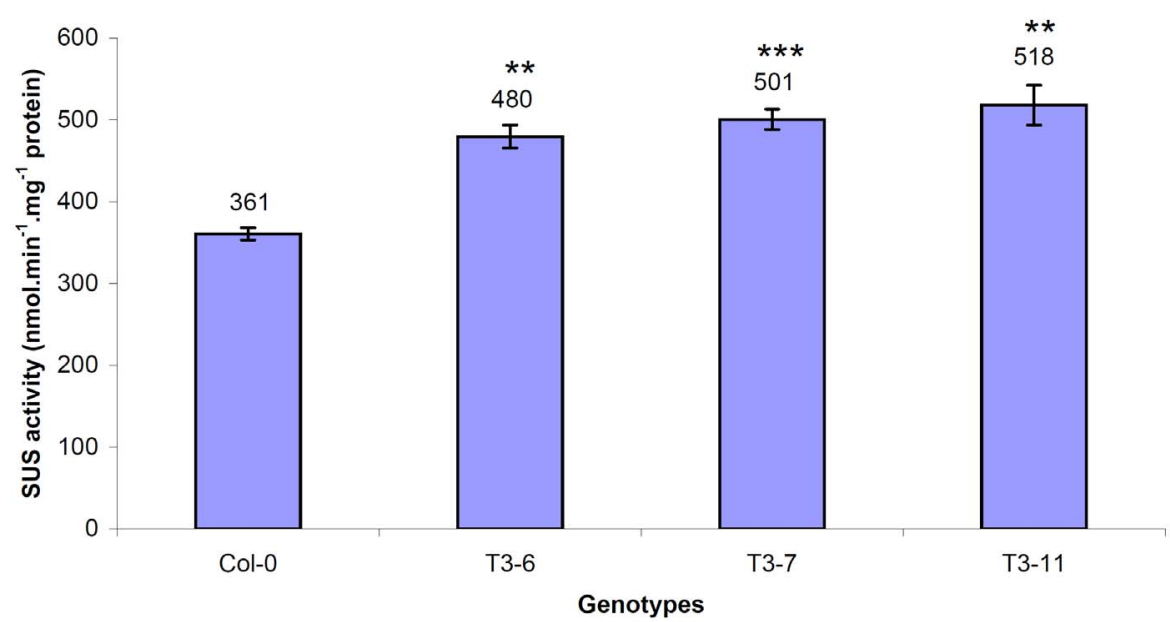

Figure 5. Sucrose synthase (SUS) activity in wild-type and three PtrSUS1 transgenic lines of Arabidopsis thaliana. SUS activity measured from the pooled young leaves and shoots of 18-d-old Arabidopsis grown in greenhouse. SUS activity was higher in transgenic plants. Error bars indicate the standard error (SE: $n=3$ ). Two asterisks **indicate very significant difference at the $99 \%$ level; three asterisks $* * *$ indicate highly significant difference at the $99.9 \%$ level.

plants.

By using both the methods, histochemical observations and biochemical measurements, we have obtained consistent results and have demonstrated the increase in SUS enzyme activity in the transgenic plants.

\subsection{Biomass Accumulation}

We further examined whether transgenic plants accumulated more biomass compared to wild type plants since they grew faster at early stage although they were phenotypically normal before bolting. Plants from three T3 transgenic lines showed highly significant increases in both fresh weights and biomass compared with the corresponding control lines (Figure 6). These increases were all statistically significant at $99.9 \%$ confidence ( $\alpha$-value of 0.001 ). Average of fresh weight from three transgenic lines was $507 \mathrm{mg} /$ plant at 4-weeks of age, corresponding to $125 \%$ of the control lines, and average of biomass of these transgenic lines was $62 \mathrm{mg} / \mathrm{plant}$, which is also $125 \%$ of controls. These data suggested that overexpression of PtrSUS1 in transgenic Arabidopsis plants resulted in increase in biomass perhaps due to increased SUS activity.

\subsection{Effects of PtrSUS1 Overexpression on Seedling Growth under Various Sucrose Concentrations}

To investigate whether PtrSUS1-overexpressing plants have elevated sucrose tolerance during seedling development, the same three transgenic lines as described above were selected for comparison in SUS enzyme activity and growth with the wild type control in response to varying concentrations of sucrose. Seeds were germinated on half-strength MS media containing different levels of sucrose in both normal light and dark conditions.

Under normal light and in the media containing 1-2\% sucrose, although visible increases of root growth from transgenic plants were observed, no highly statistical significances were found between transgenic lines and wild type (Figure 7(a)). However, with the increased sucrose concentrations, especially greater than $3 \%$, the root growth under normal light was significantly higher in transgenic than wild type plants (Figure 7(a)). It is interesting that root growth under dark condition showed statistically significant differences at all sucrose concentrations between transgenic and wild type plants (Figure 7(b)). Hypocotyls in dark showed slight or significant increase for different transgenic lines and treatments with the trend of more statistically significance found in higher sucrose concentrations (Figure 7(c)). All transgenic and wild type plants exhibited extremely slow growth in media without sucrose, indicating the importance of sucrose for the growth of young seedlings, and only growth of roots in the dark showed statistically significant differences between transgenic and control plants (Figure 7(b)). They all showed growth inhibition in media with $4-8 \%$ sucrose with the most severely reduced growth for wild type plants either in normal light or dark conditions (Figure 7). Roots of wild type were much shorter than those of three transgenic lines, and so were hypocotyls in dark (hypocotyls were too short to be measured accurately in light condition). These results indicate that overexpression of PtrSUS1 results in en- 


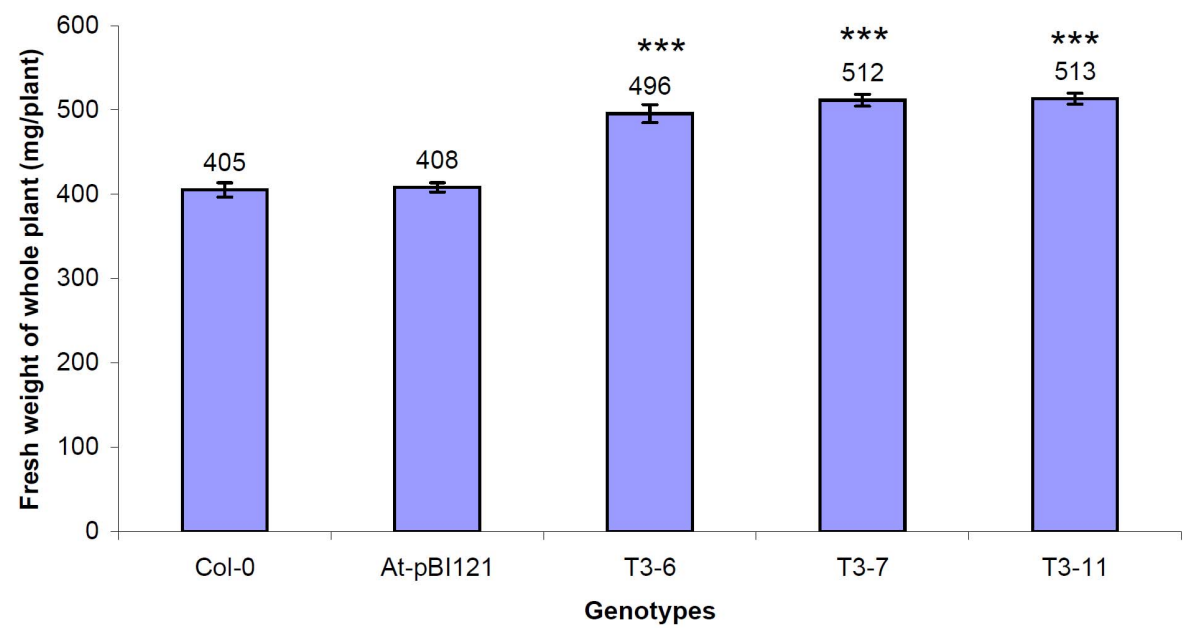

(a)

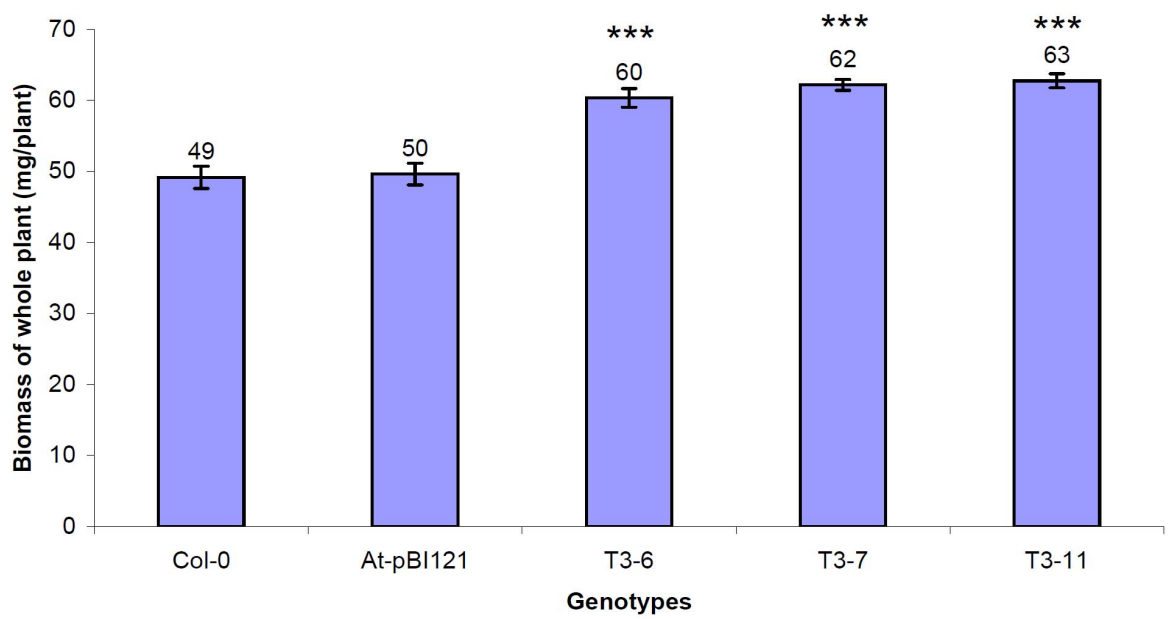

(b)

Figure 6. Fresh weight (a) and biomass of whole plant (b) for aspen SUS transgenic Arabidopsis plants grown in greenhouse. Mean \pm SE was calculated from 4-week-old 10 plants per transgenic line and wide-type (Col-0). Three asterisks ***indicate highly significant difference at the $99.9 \%$ level.

hanced seedling tolerance to higher sucrose concentrations in the growth medium.

\subsection{Effect of Constitutive PtrSUS1 Expression on SUS Activity, Fresh Weight and Biomass in Response to Different Osmotic Stresses}

To further determine the effect of PtrSUS1 transgene expression on SUS activities and correlated changes on biomass in response to different levels of sucrose, progeny of three transgenic lines and wild type plants were germinated and cultivated in vertical Petri dishes as described above under increasing serial concentrations of sucrose. As we have seen above, all transgenic and wild type Arabidopsis plants showed normal growth in media with $1-3 \%$ sucrose and displayed reduced root and hy- pocotyl length, and a general growth inhibition in response to higher sucrose concentrations from $4-8 \%$, and were unable to grow in the concentration of $10 \%$ sucrose (data not shown). These inhibitory effects increased progressively with increasing sucrose concentration, and the wild type plants reduced growth much more rapidly than transgenic plants (Figure 7). SUS enzyme activities were assayed for protein extracts of 15 -d-old three transgenic and wild type plants in response to three sucrose concentrations of 1,4 , and $8 \%$, and the results are shown in Figure 8. Overall, SUS activity increased with the elevation of sucrose concentration in growth media. Transgenic plants of T3-6 and T3-11 showed slight increase of SUS activity when growing in $1 \%$ of sucrose, and only T3-7 showed significant difference compared with wild 


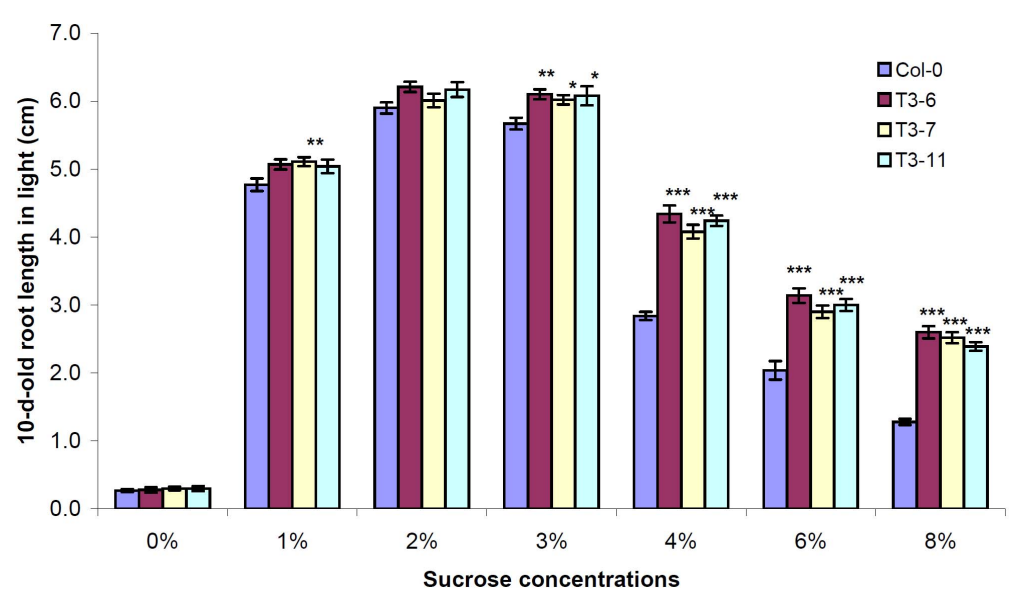

(a)

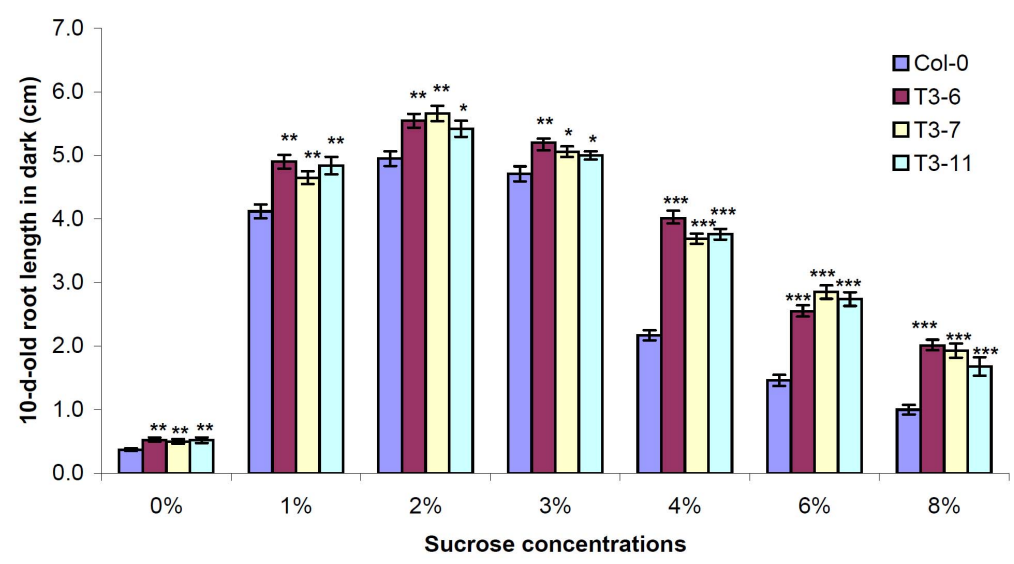

(b)

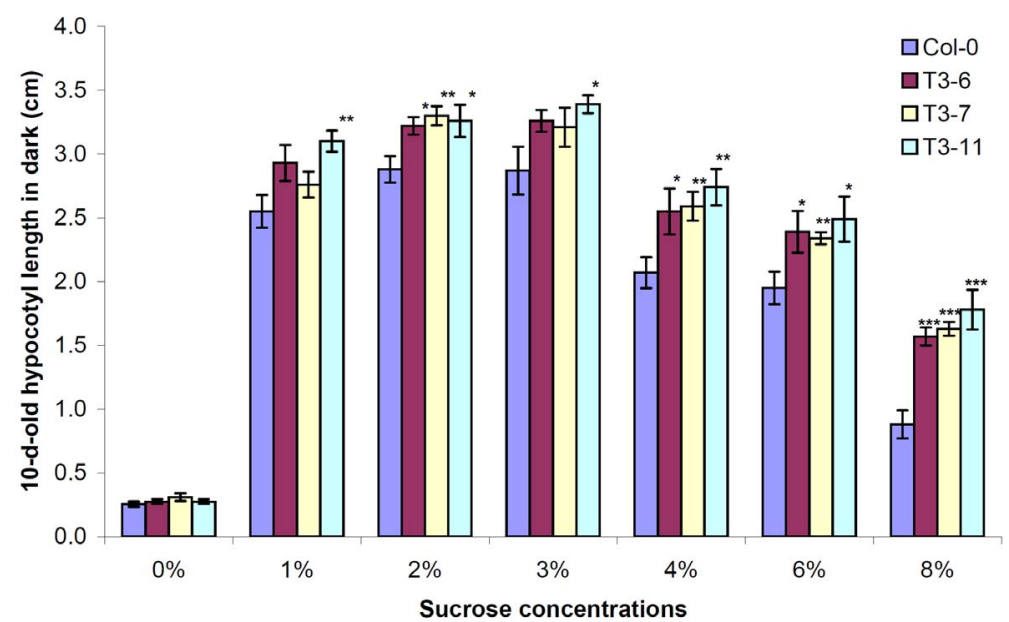

(c)

Figure 7. Root length in light condition (a), root length in dark condition (b) and hypocotyls height in dark condition (c) in transgenic Arabidopsis plants carrying and expressing aspen SUS gene in various sucrose concentrations. Mean \pm SE was calculated from 10 plants per transgenic line and wide-type (Col-0). One asterisk *indicates significant difference at the $95 \%$ level; two asterisks $* *$ indicate very significant difference at the $99 \%$ level; three asterisks $* * *$ indicate highly significant difference at the $99.9 \%$ level. 


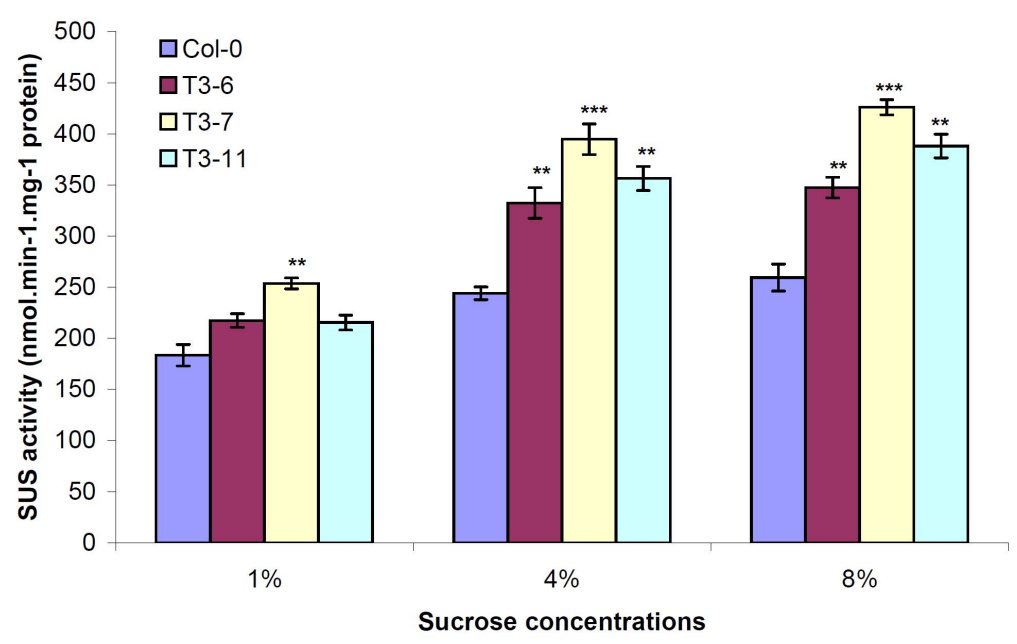

Figure 8. Comparison of SUS activity between wild-type and three PtrSUS1 transgenic lines of Arabidopsis thaliana under different sucrose concentrations. Effect of sucrose concentrations and PtrSUS1 transgene on SUS activity, measured from the 15-d-old whole plants grown in $0.5 \mathrm{x}$ MS medium in vertical Petri dish plates. SUS activity was higher in transgenic plants and treatments with higher concentrations of sucrose. Error bars indicate the standard error (SE: $n=$ $3)$. Two asterisks $* *$ indicate very significant difference at the $99 \%$ level; three asterisks $* * *$ indicate highly significant difference at the $99.9 \%$ level.

type. However, T3-6 and T3-11 showed significant increase at $99 \%$, and $\mathrm{T} 3-7$ at $99.9 \%$ confidence in response to 4 and $8 \%$ sucrose. Thus SUS activity of PtrSUS1 transgenic lines increased to a larger degree in response to higher levels of sucrose compared to that of wild type.

The above plants were also used for measuring root length in normal light in different levels of sucrose were harvested for further measuring fresh weight and biomass (dry weight) when they were 3-week-old. As shown in Figure 9, higher sucrose concentrations significantly decreased the fresh weight and dry biomass of wild type plants than at 3\% sucrose, but relatively, did not dramatically affect those of the transgenic plants. Both the fresh weight and the biomass of the transgenic plants were higher than those of the wild type plants in all concentrations of sucrose. Similarly as we have seen before, differences between transgenic and wild type plants became more drastic with the increase of sucrose especially when reaching 4\%. Statistically significant differences in fresh weight were observed for all sucrose treatments between transgenic lines and wild type plants, and so was the significant difference in biomass for most of sucrose levels except $2 \%$.

\section{DISCUSSION}

In this study, we wanted to examine the effect of the PtrSUS1 overexpression on growth and development of transgenic Arabidopsis plants. PtrSUS1 was successfully transformed into Arabidopsis plants as confirmed by genomic PCR and PtrSUS1 overexpression confirmed through RT-PCR using PtrSUS1 specific primers. Three selected transgenic lines (T3-6, T3-7 and T3-11) showed $33 \%, 39 \%$, and $43 \%$ increase, respectively, in SUS activity at the whole plant level. These results are consistent with the increase in total SUS transcript levels due to the contribution of PtSUS1 (Figure 3). This increase was sufficient to affect and change the sucrose content and metabolism of the Arabidopsis plants and thus affect the growth and development, as Coleman et al. [24] pointed out that a small increase in the expression of SUS gene caused a significant effect on carbohydrate levels.

No significantly different visible phenotypes were observed in transgenic PtrSUS1 plants compared to wild type especially after the growth for four weeks. However, the faster growth and about $40 \%$ increased biomass in transgenic plants suggested that the overexpression of PtrSUS1 gene had a significant impact on plant growth in Arabidopsis. All three transgenic lines showed significant increases in root length (dark and light conditions), hypocotyl length (dark), fresh weight and biomass compared to the wild type plants. An increase in plant height was also reported with the over-expression of SUS in poplar under the control of the cauliflower mosaic virus $35 \mathrm{~S}$ promoter [3] and in tobacco either under the single $35 \mathrm{~S}$, double $35 \mathrm{~S}$ promoter or $4 \mathrm{CL}$ promoter [24]. It is reasonably believed that the overexpression of SUS followed by the increased SUS enzyme activity enhances the capacity of plants to more effectively use photosynthates and produce more biomass. SUS has been identified as an indicator of sink strength [30], so it is possible that the greater increase of sink strength 


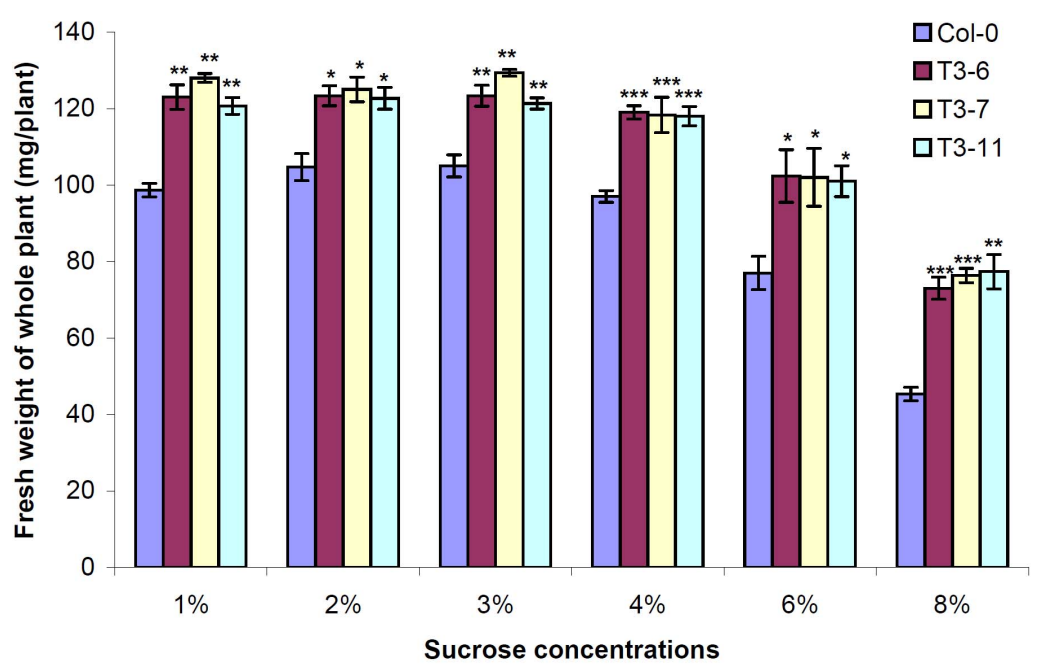

(a)

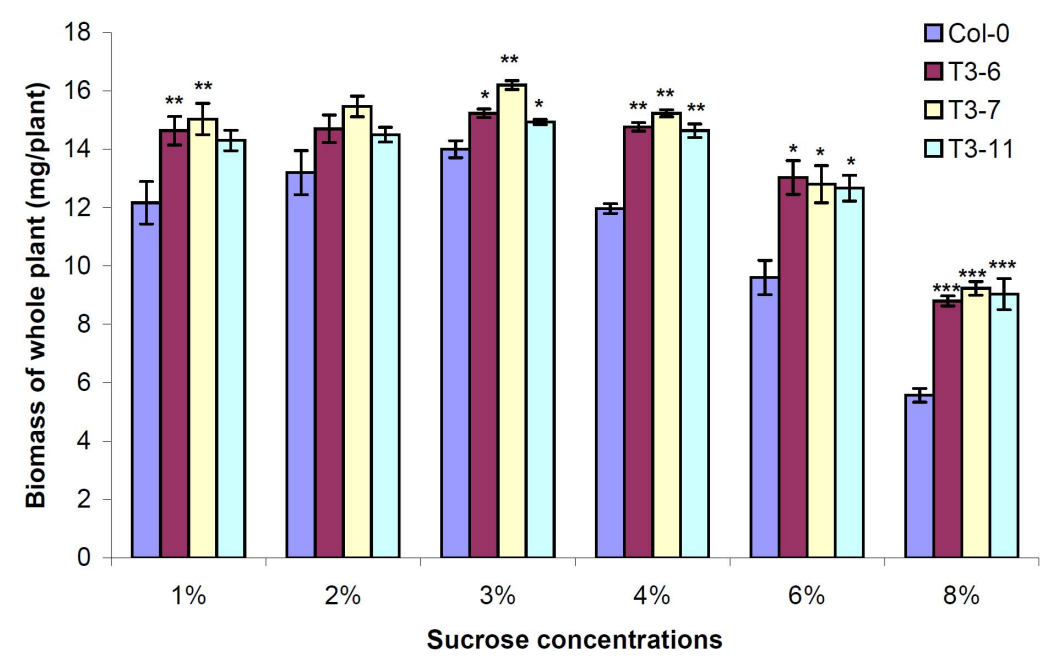

(b)

Figure 9. Fresh weight (a) and biomass of whole plant in light condition (b) for aspen SUS transgenic Arabidopsis plants in various sucrose concentrations. Mean $\pm \mathrm{SE}$ was calculated from 10 plants per transgenic line and wide-type (Col-0). One asterisk *indicates significant difference at the $95 \%$ level; two asterisks **indicate very significant difference at the $99 \%$ level; three asterisks $* * *$ indicate highly significant difference at the $99.9 \%$ level.

caused by the overexpression of SUS promotes plant growth. This suggests the possibility of increasing convertible biomass for biofuel resources through manipulating SUS gene.

There exist some controversy about the roles of individual SUS in the biosynthesis of starch and cellulose, and plant growth and development. In the suppression studies of SUS gene expression and enzyme activity, a decrease in starch and/or cellulose content has been demonstrated in potato [1,17], in maize [38], in carrot $[39,40]$, in pea [41], and in cotton [31]. In contrast, the lack of phenotypic changes in the single and multiple knockout lines of AtSUS1, AtSUS2, AtSUS3, and AtSUS4 isoforms in Arabidopsis [42] has brought uncertainty whether this enzyme is necessary in starch and cellulose biosynthesis. This could be due to plasticity in plant metabolism and utilization of alternative pathways of sucrose cleavage such as invertase, the transcript of which is very high in Arabidopsis sink tissues [43]. Reverse genetics approaches, hence, should be further used to provide more and definitive evidence for the importance of SUS in Arabidopsis. On the other hand, the results from the studies of SUS upregulation are not consistent. The over-expression of cotton SUS in tobacco showed an 
increase in starch accumulation in transgenic lines with the promoter of 4CL but no significant increase was observed in other lines with $35 \mathrm{~S}$ promoter [24]. In the same experiment, only one transgenic line with the double $35 \mathrm{~S}$ promoter showed a significant increase in cellulose content.

In this study, we also observed that PtrSUS1 transgenic Arabidopsis plants have the feature of early bolting and flowering compared with wild type. The role of SUS in reproductive development has been verified from several studies. SUS activity was severely inhibited by the antisense RNA in flowers and fruit pericarp tissues in antisense transgenic tomato plants, and in turn causing a reduced fruit set and a slower growth rate of fruits [30]. These results suggest that SUS participates in regulating sucrose import capacity of young tomato fruit, which is a determinant for fruit set and development. RNAi approach was successfully used to silence the expression of SUS gene in the endosperm and led to the arrest of early seed development in cotton [44]. While studying the differential expression of three SUS genes during fruit development in citrus, Komatsu et al. [45] reported that SUS activity in edible tissue was high in the early stages and decreased until mid-development, then rapidly increased during maturation. The increase in activity during maturation paralleled that of sucrose accumulation, indicating the important role of SUS on sugar metabolism when sucrose is accumulated in fruit. Based on enzyme activity, transcript levels, and protein localization, the importance of SUS for sucrose metabolism has been demonstrated in starch-storing reproductive structures of legumes such as Vicia [46] and Pisum sp. [41], in developing nectaries of ornamental tobacco [47], and in cotton seeds $[19,48]$. High SUS activity was also found in reproductive sink tissues of several other plant species including Arabidopsis silique [5], canola seed and silique [49], cotton fiber and seed [49,50], wheat grain [51], rice [52], and tomato fruit [18]. High SUS activity in these reproductive tissues suggests an important role of SUS enzyme for sucrose metabolism in determining sink strength. Fallahi et al. [5] pointed out that during early stages of seed development, high SUS activity is needed to establish a strong sink for sucrose by breakdown of imported sucrose to fructose and UDP-glucose.

The observed increased tolerance to higher sucrose stress of the PtrSUS1 transgenic plants were positively correlated with the increased SUS activities. We showed here that, under high sucrose stress, transgenic plants over-expressing PtrSUS1 cleaved more sucrose, as deduced from their higher root and hypocotyls growth, and higher fresh weight and biomass per plant in comparison with the wild type plants (Figure 5, 7, and 9). This increased tolerance to higher sucrose or lower osmotic pressure is closely related to the over-expression of PtrSUS1 and correspondingly increased SUS activities in these transgenic Arabidopsis lines, suggesting the role of SUS in cleaving sucrose. This increase is further strengthened because the SUS expression was upregulated by osmotic stress rather than by the increase in sucrose concentration per se $[53,54]$. Moreover, recent studies also suggest that AtSUS1 gene is regulated by sugars (sucrose, glucose, and mannose) through hexokinase-dependent mechanisms [55]. Sugars are known to be inducers/repressors of gene expression and they have key roles in signal transduction pathways [56,57]. As a conclusion, the regulation of AtSUS1 possibly involves two or more transduction pathways depending on the nature and strength of the signal [55].

In summary, the current work provided new information to advance our overall knowledge about the effect of heterologous SUS on transgenic Arabidopsis plants and their responses to various concentrations of sucrose. We demonstrated here that the over-expression of an aspen SUS (PtrSUS1) alone in Arabidopsis enhanced SUS activity, indicating that the foreign SUS gene functions in a similar manner to the endogenous AtSUS activity. The increased SUS activity in turn changed sucrose metabolism inside the transgenic plants. The observed phenotypes with faster growth and early flowering as well as tolerance-enhanced in high sucrose are closely correlated with the increased cleavage capacity of SUS for sucrose in plants. The present results emphasize the importance of SUS-mediated sucrose cleavage process in response to high sucrose concentrations, and provide a basis to better understanding of the integrated functions of SUS during plant growth and development.

\section{ACKNOWLEDGEMENTS}

This work was supported by a grant from National Science Foundation's CAREER program (IBN- 0236492). This work was also partially supported by the World Class University project of the Ministry of Science and Technology of Korea (R31-2009-000-20025-0). We wish to thank Sandra Hubscher and Rama Joshi for critical reading of this manuscript.

\section{REFERENCES}

[1] Munoz, F.J., Baroja-Fernandez, E., Moran-Zorzano, M.T., Viale, A.M., Etxeberria, E., Alonso-Casajus, N., et al. (2005) Sucrose synthase controls both intracellular ADP glucose levels and transitory starch biosynthesis in source leaves. Plant Cell Physiol, 46(8), 1366-1376.

[2] Baroja-Fernandez, E., Munoz, F.J., Saikusa, T., Rodriguez-Lopez, M., Akazawa, T. and Pozueta-Romero, J. (2003) Sucrose synthase catalyzes the de novo production of ADPglucose linked to starch biosynthesis in heterotrophic tissues of plants. Plant Cell Physiol, 44(5), 
500-509.

[3] Konishi, T., Ohmiya, Y. and Hayashi, T. (2004) Evidence that sucrose loaded into the phloem of a poplar leaf is used directly by sucrose synthase associated with various beta-glucan synthases in the stem. Plant Physiol, 134(3), 1146-1152.

[4] Klotz, K.L. and Haagenson, D.M. (2008) Wounding, anoxia and cold induce sugarbeet sucrose synthase transcriptional changes that are unrelated to protein expression and activity. J Plant Physiol, 165(4), 423-434.

[5] Fallahi, H., Scofield, G.N., Badger, M.R., Chow, W.S., Furbank, R.T. and Ruan, Y.L. (2008) Localization of sucrose synthase in developing seed and siliques of Arabidopsis thaliana reveals diverse roles for SUS during development. J Exp Bot, 59(12), 3283-3295.

[6] Winter, H., Huber, J.L. and Huber, S.C. (1997) Membrane association of sucrose synthase: changes during the graviresponse and possible control by protein phosphorylation. Febs Letters, 420(2-3), 151-155.

[7] Matic, S., Akerlund, H.E., Everitt, E. and Widell, S. (2004) Sucrose synthase isoforms in cultured tobacco cells. Plant Physiology and Biochemistry, 42(4), 299-306.

[8] Winter, H., Huber, J.L. and Huber, S.C. (1998) Identification of sucrose synthase as an actin-binding protein. Febs Letters, 430(3), 205-208.

[9] Etxeberria, E. and Gonzalez, P. (2003) Evidence for a tonoplast-associated form of sucrose synthase and its potential involvement in sucrose mobilization from the vacuole. Journal of Experimental Botany, 54(386), 1407-1414.

[10] Carlson, S.J. and Chourey, P.S. (1996) Evidence for plasma membrane-associated forms of sucrose synthase in maize. Molecular \& General Genetics, 252(3), 303-10.

[11] Hardin, S.C., Duncan, K.A. and Huber, S.C. (2006) Determination of structural requirements and probable regulatory effectors for membrane association of maize sucrose synthase. Plant Physiology, 141(3), 1106-1119.

[12] Amor, Y., Haigler, C.H., Johnson, S., Wainscott, M. and Delmer, D.P. (1995) A Membrane-Associated Form of Sucrose Synthase and Its Potential Role in Synthesis of Cellulose and Callose in Plants. Proceedings of the $\mathrm{Na}$ tional Academy of Sciences of the United States of America, 92(20), 9353-9357.

[13] Salnikov, V.V., Grimson, M.J., Delmer, D.P. and Haigler, C.H. (2001) Sucrose synthase localizes to cellulose synthesis sites in tracheary elements. Phytochemistry, 57(6), 823-833.

[14] Salnikov, V.V., Grimson, M.J., Seagull, R.W. and Haigler, C.H. (2003) Localization of sucrose synthase and callose in freeze-substituted secondary-wall-stage cotton fibers. Protoplasma, 221(3-4), 175-184.

[15] Albrecht, G. and Mustroph, A. (2003) Localization of sucrose synthase in wheat roots: increased in situ activity of sucrose synthase correlates with cell wall thickening by cellulose deposition under hypoxia. Planta, 217(2), 252-260.

[16] Albrecht, G. and Mustroph, A. (2003) Sucrose utilization via invertase and sucrose synthase with respect to accumulation of cellulose and callose synthesis in wheat roots under oxygen deficiency. Russian Journal of Plant Physiology, 50(6), 813-820.

[17] Zrenner, R., Salanoubat, M., Willmitzer, L. and Sonne- wald, U. (1995) Evidence of the crucial role of sucrose synthase for sink strength using transgenic potato plants (Solanum tuberosum L.). Plant J, 7(1), 97-107.

[18] Sun, J.D., Loboda, T., Sung, S.J.S. and Black, C.C. (1992) Sucrose Synthase in Wild Tomato, LycopersiconChmielewskii, and Tomato Fruit Sink Strength. Plant Physiology, 98(3), 1163-1169.

[19] Ruan, Y.L., Chourey, P.S., Delmer, D.P. and Perezgrau, L. (1997) The differential expression of sucrose synthase in relation to diverse patterns of carbon partitioning in developing cotton seed. Plant Physiology, 115(2), 375-85.

[20] Heinlein, M. and Starlinger, P. (1989) Tissue-Specific and Cell-Specific Expression of the 2 Sucrose Synthase Isoenzymes in Developing Maize Kernels. Molecular \& General Genetics, 215(3), 441-446.

[21] Wittich, P.E. and Vreugdenhil, D. (1998) Localization of sucrose synthase activity in developing maize kernels by in situ enzyme histochemistry. Journal of Experimental Botany, 49(324), 1163-1171.

[22] Dejardin, A., Rochat, C., Wuilleme, S. and Boutin, J.P. (1997) Contribution of sucrose synthase, ADP-glucose pyrophosphorylase and starch synthase to starch synthesis in developing pea seeds. Plant Cell and Environment, 20(11), 1421-1430.

[23] Dejardin, A., Rochat, C., Maugenest, S. and Boutin, J.P. (1997) Purification, characterization and physiological role of sucrose synthase in the pea seed coat (Pisum sativum L). Planta, 201(2), 128-137.

[24] Coleman, H.D., Ellis, D.D., Gilbert, M. and Mansfield, S.D. (2006) Up-regulation of sucrose synthase and UDP-glucose pyrophosphorylase impacts plant growth and metabolism. Plant Biotechnol J, 4(1), 87-101.

[25] Chopra, J., Kaur, N. and Gupta, A.K. (2007) Sustained activities of carbon metabolizing enzymes determine seed size in Vigna radiata (mungbean). Current Science, 92(10), 1420-1424.

[26] Chopra, J., Kaur, N. and Gupta, A. (2005) Role of enzymes of sucrose-starch conversion in seed sink strength in mung bean. Biologia Plantarum, 49(4), 561-566.

[27] Chopra, J., Kaur, N. and Gupta, A.K. (2000) Ontogenic changes in enzymes of carbon metabolism in relation to carbohydrate status in developing mungbean reproducetive structures. Phytochemistry, 53(5), 539-548.

[28] Ito, A., Hayama, H. and Kashimura, Y. (2002) Sugar metabolism in buds during flower bud formation: a comparison of two Japanese pear [Pyrus pyrifolia (Burm.) Nak.] cultivars possessing different flowering habits. Scientia Horticulturae, 96(1-4), 163-175.

[29] Coleman, H.D., Yan, J. and Mansfield, S.D. (2009) Sucrose synthase affects carbon partitioning to increase cellulose production and altered cell wall ultrastructure. Proc Natl Acad Sci U S A, 106(31), 13118-13123.

[30] D'aoust, M.A., Yelle, S. and Nguyen-Quoc, B. (1999) Antisense inhibition of tomato fruit sucrose synthase decreases fruit setting and the sucrose unloading capacity of young fruit. Plant Cell, 11(12), 2407-2418.

[31] Ruan, Y.L., Llewellyn, D.J. and Furbank, R.T. (2003) Suppression of sucrose synthase gene expression represses cotton fiber cell initiation, elongation, and seed development. Plant Cell, 15(4), 952-964.

[32] Meng M., Geisler M., Johansson H., Mellerowicz E.J., Karpinski S., and Kleczkowski LA. (2007) Differential 
tissue/organ-dependent expression of two sucrose- and cold-responsive genes for UDP-glucose pyrophosphorylase in Populus. Gene 389(2), 186-195.

[33] Clough, S.J. and Bent, A.F. (1998) Floral dip: a simplified method for Agrobacterium-mediated transformation of Arabidopsis thaliana. Plant $J$, 16(6), 735-743.

[34] Sergeeva, L.I., Vonk, J., Keurentjes, J.J., Van Der Plas, L.H., Koornneef, M. and Vreugdenhil, D. (2004) Histochemical analysis reveals organ-specific quantitative trait loci for enzyme activities in Arabidopsis. Plant Physiol, 134(1), 237-245.

[35] Xu, D.P., Sung, S.J., Loboda, T., Kormanik, P.P. and Black, C.C. (1989) Characterization of Sucrolysis via the Uridine Diphosphate and Pyrophosphate-Dependent $\mathrm{Su}-$ crose Synthase Pathway. Plant Physiol, 90(2), 635-642.

[36] Sergeeva, L.I. and Vreugdenhil, D. (2002) In situ staining of activities of enzymes involved in carbohydrate metabolism in plant tissues. J Exp Bot, 53(367), 361-370.

[37] Ruan, Y.L., Xu, S.M., White, R. and Furbank, R.T. (2004) Genotypic and developmental evidence for the role of plasmodesmatal regulation in cotton fiber elongation mediated by callose turnover. Plant Physiology, 136(4), 4104-4113.

[38] Chourey, P.S. and Nelson, O.E. (1976) The enzymatic deficiency conditioned by the shrunken-1 mutations in maize. Biochem Genet, 14(11-12), 1041-1055.

[39] Tang, G.Q. and Sturm, A. (1999) Antisense repression of sucrose synthase in carrot (Daucus carota L.) affects growth rather than sucrose partitioning. Plant Mol Biol, 41(4), 465-479.

[40] Sturm, A. and Tang, G.Q. (1999) The sucrose-cleaving enzymes of plants are crucial for development, growth and carbon partitioning. Trends Plant Sci, 4(10), 401-7.

[41] Barratt, D.H., Barber, L., Kruger, N.J., Smith, A.M., Wang, T.L. and Martin, C. (2001) Multiple, distinct isoforms of sucrose synthase in pea. Plant Physiol, 127(2), 655-664.

[42] Bieniawska, Z., Paul Barratt, D.H., Garlick, A.P., Thole, V., Kruger, N.J., Martin, C., et al. (2007) Analysis of the sucrose synthase gene family in Arabidopsis. Plant $J$, 49(5), 810-828.

[43] Tymowska-Lalanne, Z. and Kreis, M. (1998) Expression of the Arabidopsis thaliana invertase gene family. Planta, 207(2), 259-265.

[44] Ruan, Y.L., Llewellyn, D.J., Liu, Q., Xu, S.M., Wu, L.M., Wang, L., et al. (2008) Expression of sucrose synthase in the developing endosperm is essential for early seed development in cotton. Functional Plant Biology, 35(5), 382-293.

[45] Komatsu, A., Moriguchi, T., Koyama, K., Omura, M. and
Akihama, T. (2002) Analysis of sucrose synthase genes in citrus suggests different roles and phylogenetic relationships. J Exp Bot, 53(366), 61-71.

[46] Heim, U., Weber, H., Baumlein, H. and Wobus, U. (1993) A sucrose-synthase gene of Vicia faba L.: expression pattern in developing seeds in relation to starch synthesis and metabolic regulation. Planta, 191(3), 394-401.

[47] Ren, G., Healy, R.A., Horner, H.T., James, M.G. and Thornburg, R.W. (2007) Expression of starch metabolic genes in the developing nectaries of ornamental tobacco plants. Plant Science, 173(6), 621-637.

[48] Ruan, Y.L., Llewellyn, D.J. and Furbank, R.T. (2000) Pathway and control of sucrose import into initiating cotton fibre cells. Australian Journal of Plant Physiology, 27(8-9), 795-800.

[49] King, S.P., Lunn, J.E. and Furbank, R.T. (1997) Carbohydrate Content and Enzyme Metabolism in Developing Canola Siliques. Plant Physiol, 114(1), 153-160.

[50] Ruan, Y.L. and Chourey, P.S. (1998) A fiberless seed mutation in cotton is associated with lack of fiber cell initiation in ovule epidermis and alterations in sucrose synthase expression and carbon partitioning in developing seeds. Plant Physiol, 118(2), 399-406.

[51] Dale, E.M. and Housley, T.L. (1986) Sucrose Synthase Activity in Developing Wheat Endosperms Differing in Maximum Weight. Plant Physiol, 82(1), 7-10.

[52] Chan, H.Y., Ling, T.Y., Juang, R.H., Ting, I.N., Sung, H.Y. and Su, J.C. (1990) Sucrose Synthase in Rice Plants: Growth-Associated Changes in Tissue Specific Distributions. Plant Physiol, 94(3), 1456-1461.

[53] Baud, S., Vaultier, M.N. and Rochat, C. (2004) Structure and expression profile of the sucrose synthase multigene family in Arabidopsis. J Exp Bot, 55(396), 397-409.

[54] Dejardin, A., Sokolov, L.N. and Kleczkowski, L.A. (1999) Sugar/osmoticum levels modulate differential abscisic acid-independent expression of two stress-responsive sucrose synthase genes in Arabidopsis. Biochem J, 344(2), 503-509.

[55] Ciereszkoa, I. and Kleczkowski, L.A. (2002) Glucose and mannose regulate the expression of a major sucrose synthase gene in Arabidopsis via hexokinase-dependent mechanisms. Plant Physiology and Biochemistry, 40(11), 907-911.

[56] Koch, K.E. (1996) Carbohydrate-Modulated Gene Expression in Plants. Annu Rev Plant Physiol Plant Mol Biol, 47, 509-540.

[57] Pego, J.V., Kortstee, A.J., Huijser, C. and Smeekens, S.C. (2000) Photosynthesis, sugars and the regulation of gene expression. J Exp Bot, 51 Spec No, 407-416. 